\title{
Correction to: Transethnic insight into the genetics of glycaemic traits: fine-mapping results from the Population Architecture using Genomics and Epidemiology (PAGE) consortium
}

\author{
Stephanie A. Bien ${ }^{1} \cdot$ James S. Pankow ${ }^{2} \cdot$ Jeffrey Haessler $^{1} \cdot$ Yinchang Lu $^{3}$. \\ Nathan Pankratz ${ }^{4}$ - Rebecca R. Rohde ${ }^{5}$ - Alfred Tamuno ${ }^{6}$ - Christopher S. Carlson ${ }^{1}$. \\ Fredrick R. Schumacher ${ }^{7} \cdot$ Petra Bůžková $^{8}$ - Martha L. Daviglus ${ }^{9}$ - Unhee Lim ${ }^{10}$. \\ Myriam Fornage $^{11}$ - Lindsay Fernandez-Rhodes ${ }^{5}$ - Larissa Avilés-Santa ${ }^{12}$. \\ Steven Buyske $^{13,14}$ • Myron D. Gross ${ }^{4}$ - Mariaelisa Graff ${ }^{5}$ - Carmen R. Isasi $^{15}$. $^{1}$ \\ Lewis H. Kuller ${ }^{16}$ • JoAnn E. Manson ${ }^{17}$ • Tara C. Matise ${ }^{13}$ - Ross L. Prentice ${ }^{1}$. \\ Lynne R. Wilkens $^{10}$ • Sachiko Yoneyama ${ }^{18,19}$ • Ruth J. F. Loos ${ }^{6,20,21,22}$. \\ Lucia A. Hindorff ${ }^{23}$ • Loic Le Marchand ${ }^{10}$ • Kari E. North ${ }^{5,24}$. \\ Christopher A. Haiman ${ }^{25}$ - Ulrike Peters ${ }^{1}$ • Charles Kooperberg ${ }^{1}$
}

Published online: 16 October 2017

(C) Springer-Verlag GmbH Germany 2017

\section{Correction to: Diabetologia}

https://doi.org/10.1007/s00125-017-4405-1

The authors regret that Yinchang Lu's name incorrectly included a middle initial in the author list. The details given in this erratum are correct.

The online version of the original article can be found at https://oi.org/ 10.1007/s00125-017-4405-1

Stephanie A. Bien

sbien@fredhutch.org

1 Division of Public Health Sciences, Fred Hutchinson Cancer Research Center, 1100 Fairview Ave N., Seattle, WA 98109-1024, USA

2 Division of Epidemiology and Community Health, University of Minnesota, Minneapolis, MN, USA

3 Department of Biological Sciences, Vanderbilt University, Nashville, TN, USA

4 Department of Laboratory Medicine and Pathology, University of Minnesota, Minneapolis, MN, USA

5 Department of Epidemiology, School of Public Health, University of North Carolina at Chapel Hill, Chapel Hill, NC, USA
6 The Department of Preventive Medicine, The Icahn School of Medicine at Mount Sinai, New York, NY, USA

7 Department of Epidemiology and Biostatistics, Case Western Reserve University, Cleveland, OH, USA

8 Department of Biostatistics, University of Washington, Seattle, WA, USA

9 Department of Medicine, Institute for Minority Health Research, University of Illinois at Chicago, Chicago, IL, USA

10 Epidemiology Program, University of Hawaii Cancer Center, Honolulu, HI, USA

11 Human Genetics Center, University of Texas Health Science Center at Houston, Houston, TX, USA

12 Division of Cardiovascular Sciences, National Heart, Lung, and Blood Institute, National Institutes of Health, Bethesda, MD, USA 
13 Department of Genetics, Rutgers University, Piscataway, NJ, USA

14 Department of Statistics, Rutgers University, Newark, NJ, USA

15 Department of Epidemiology \& Population Health, Albert Einstein College of Medicine, Bronx, NY, USA

16 Department of Epidemiology, University of Pittsburgh, Pittsburgh, PA, USA

17 Department of Epidemiology, Harvard T.H. Chan School of Public Health, Boston, MA, USA

18 Department of Ophthalmology and Visual Sciences, University of Michigan, Ann Arbor, MI, USA

19 Department of Epidemiology, University of Michigan, Ann Arbor, MI, USA

20 MRC Epidemiology Unit, Institute of Metabolic Science, University of Cambridge, Cambridge, UK

21 The Charles Bronfman Institute for Personalized Medicine, The Icahn School of Medicine at Mount Sinai, New York, NY, USA

22 The Icahn School of Medicine at Mount Sinai, New York, NY, USA

23 National Human Genome Research Institute, National Institutes of Health, Bethesda, MD, USA

24 Carolina Center for Genome Sciences, University of North Carolina at Chapel Hill, Chapel Hill, NC, USA

25 Department of Preventive Medicine, Keck School of Medicine, University of Southern California/Norris Comprehensive Cancer Center, Los Angeles, CA, USA 